Momigliano, Ausgewählte Schriften, Band 2 


\title{
Arnaldo Momigliano
}

\section{Ausgewählte Schriften zur Geschichte und Geschichtsschreibung}

\author{
Herausgegeben von Glenn W. Most \\ unter Mitwirkung von \\ Wilfried Nippel und Anthony Grafton
}




\title{
Arnaldo Momigliano
}

\section{Ausgewählte Schriften zur Geschichte und Geschichtsschreibung}

\author{
Band 2 \\ Spätantike bis Spätaufklärung
}

\author{
Herausgegeben \\ von Anthony Grafton \\ Übersetzt von Kai Brodersen \\ und Andreas Wittenburg
}

Verlag J. B. Metzler

Stuttgart - Weimar 
Die Texte 1, 3, 5 und 9 hat Andreas Wittenburg, die restlichen Texte sowie die Anmerkungen zu Text 1 hat Kai Brodersen übersetzt.

Die Deutsche Bibliothek - CIP-Einheitsaufnahme

\title{
Momigliano, Arnaldo:
}

Ausgewählte Schriften zur Geschichte und Geschichtsschreibung /

Arnaldo Momigliano. Hrsg. von Glenn W. Most unter Mitw. von Wilfried Nippel und Anthony Grafton. - Stuttgart ; Weimar : Metzler ISBN 978-3-476-01514-3

Bd. 2. Spätantike bis Spätaufklärung / hrsg. von Anthony Grafton. Übers. von Kai Brodersen und Andreas Wittenburg. - 1999 ISBN 978-3-476-01512-9

\author{
ISBN 978-3-476-01514-3 (Gesamtwerk) \\ ISBN 978-3-476-01512-9 \\ ISBN 978-3-476-03683-4 (eBook) \\ DOI 10.1007/978-3-476-03683-4
}

Dieses Werk einschließlich aller seiner Teile ist urheberrechtlich geschützt. Jede Verwertung außerhalb der engen Grenzen des Urheberrechtsgesetzes ist ohne Zustimmung des Verlages unzulässig und strafbar. Das gilt insbesondere

für Vervielfältigungen, Übersetzungen, Mikroverfilmungen und die

Einspeicherung und Verarbeitung in elektronischen Systemen.

(C) 1999 Springer-Verlag GmbH Deutschland

Ursprünglich erschienen bei J. B. Metzlersche Verlagsbuchhandlung und Carl Ernst Poeschel Verlag GmbH in Stuttgart 1999 


\section{Inhalt}

1. Alte Geschichte und antiquarische Forschung 1

2. Cassiodor und die italienische Kultur seiner Zeit 37

3. Bemerkungen über die Legende vom Christentum Senecas 59

4. Die Wiederentdeckung des Polybios im europäischen Westen 79

5. Herodot und die moderne Geschichtsschreibung 101

6. Der erste politische Kommentar zu Tacitus 115

7. Die Einrichtung der Geschichte als akademisches Fach und ihre Implikationen 135

8. Die Schüler Mabillons in Italien 155

9. Die klassischen Studien des Scipione Maffei 175

10. Römische Hünen und Helden in Vicos Scienza Nuova 195

11. Ein Vorspiel zu Gibbon im 18. Jahrhundert 221

12. Gibbons Beitrag zur Historischen Methode 237

$\begin{array}{ll}\text { Anmerkungen } & 257\end{array}$

Verzeichnis der Erstpublikationen 319 


\section{Einleitung}

Arnaldo Momigliano hat stets prinzipiell darauf bestanden, daß er sich auf die Wissenschaftsgeschichte und die Geschichte der klassischen Tradition zwischen der Spätantike und der frühen Neuzeit nie wirklich spezialisiert habe: er sei vielmehr ein einfacher Althistoriker, der sich am liebsten mit der Analyse ganz normaler historischer Texte und Probleme der Antike befasse. In seinen Vorträgen und Aufsätzen ${ }^{1}$ zitierte er ständig mit großem Respekt die Untersuchungen von Freunden und Kollegen, die sich seines Erachtens auf diesem Gebiet wesentlich besser auskannten als er selbst. Im übrigen riet er jüngeren Wissenschaftlern immer wieder davon $a b$, eine historiographische Untersuchung vorzunehmen bzw. eine solche Dissertation zu schreiben. Seiner Meinung nach vermochte nur ein erfahrener Althistoriker oder Altphilologe, der sich mit einem bestimmten klassischen Text oder Problemkreis über Jahre hinweg wissenschaftlich auseinandergesetzt hatte, die Entwicklung der vorangegangenen Forschung oder die Überlieferung der klassischen Texte nachzuzeichnen. Einerseits sei »das Gros der Doktoranden " - so Momigliano in einer für ihn typischen ironischen Wendung -, das in der Philologie nicht bewandert sei, kaum in der Lage, das Nachleben der Antike als Thema zu wählen. ${ }^{2}$ Andererseits lieferten die meisten Geschichtswissenschaftler und Literaturhistoriker, die versuchten, die Arbeit eines Philologen oder eines Historikers historisch zu analysieren, nur den schlagenden Beweis dafür, daß sie weder den historischen Kontext noch den Inhalt der betreffenden Texte verstanden hatten. ${ }^{3}$ An etlichen wissenschaftsgeschichtlichen Monographien übte er schonungslose Kritik. Fast könnte man annehmen, daß Momigliano seine Leser vor allem davon abhalten wollte, seinen besonderen historiographischen Ansatz nachzuahmen.

Dennoch wurden etliche wissenschaftsgeschichtliche Untersuchungen Momiglianos im Laufe der Zeit zu Klassikern der historiographischen Literatur; sie wurden zumal von amerikanischen und englischen, italienischen und französischen Gelehrten in fast allen Fächern der Geisteswissenschaften mit Interesse und Gewinn gelesen. ${ }^{4}$ Mehr als einmal gelang es Momigliano, die Aufmerksamkeit der Spezialisten 
für die Geistesgeschichte des Mittelalters oder der Renaissance auf wichtige Themen zu lenken, die wissenschaftlich in Vergessenheit geraten waren. In den letzten zwanzig Jahren sind die Geschichte der antiquarischen Studien und die Entwicklung des Museums zwischen Renaissance und Barock zu einem Lieblingsthema der historischen wie der kunsthistorischen Forschung geworden. ${ }^{5}$ Noch heute stellt Momiglianos Essay von 1950, ^Ancient History and the Antiquarian « (unten, Text 1) - nach dem Urteil von Francis Haskell - die mit Abstand beste Zusammenfassung und Analyse dieser Tradition der Studien und Institutionen dar. ${ }^{6}$ Jeder Historiker, der sich für die Versuche der Geschichtsschreiber des 16. und 17. Jahrhunderts interessiert, die Gesellschaften der Neuen Welt zu schildern, weiß, daß Herodot in diesem Zusammenhang eine wichtige Rolle gespielt hat. Dessen Geschichte der Kriege zwischen Griechen und Persern wurde im 16. Jahrhundert zum Muster der eingehenden Untersuchung einer fremden Gesellschaft, das von Historikern und Autoren von Reiseberichten immer wieder nachgeahmt wurde. Seit Ranke war allgemein anerkannt, daß Thukydides die Tradition der diachronen, politischen und militärischen Geschichtsschreibung begründet hatte. ${ }^{7}$ Momigliano kommt das Verdienst zu, in jener anderen Tradition der synchronen, ethnographischen Geschichte, die sich von Herodot herleitet, den Ursprung der frühneuzeitlichen Anthropologie entdeckt zu haben. ${ }^{8}$

Momigliano ist es nie gelungen, die Erträge seiner Forschungen in einer umfassenden Synthese darzulegen. Schon 1955 begann er, seine Artikel, die in zahlreichen Zeitschriften und Festschriften verstreut waren, in einer Reihe von Sammelbänden, seinen Contributi, nachzudrucken. 1962 hielt er die "Sather Lectures« an der University of California at Berkeley. Vor einem großem Publikum wagte er den Versuch, die wichtigsten Traditionen der klassischen Geschichtsschreibung zu rekonstruieren. Zwar wurden Momiglianos Vorträge, die einen nachhaltigen Eindruck auf die Historiker und Anthropologen unter seinen Zuhörern machten, zu einem großen Erfolg, doch gab sich Momigliano mit keiner der vielen Fassungen des Textes zufrieden, die er über die Jahrzehnte hinweg schrieb und revidierte. Erst nach seinem Tode ist das Buch von Riccardo di Donato herausgegeben worden. ${ }^{9}$ Jüngere Historiker hatten es also nicht leicht, den Weg zu Momigliano zu finden. Paradoxerweise gelang es ihm dennoch immer wieder, Wissenschaftler aus fast aller Herren Länder an genau die Probleme und Themen heranzuführen, die er als besonders gefährlich für sie betrachtete. Wider Willen ist Momigliano heute fast Mode geworden, und zwar nicht nur in der 
Alten Geschichte, sondern auch in anderen Disziplinen. Will man auf überzeugende Weise erklären, wie es dazu gekommen ist, daß dieser Althistoriker einen so großen Einfluß auf Mediävisten und Renaissanceforscher, Kunsthistoriker und Literaturwissenschaftler auszuüben vermochte, so muß man die Entwicklung seiner Interessen verfolgen.

Unter italienischen Gelehrten gibt es des öfteren solche, die im Alter von achtzehn oder zwanzig Jahren die ersten Erträge ihrer Forschungen veröffentlichen. Aber selbst für italienische Verhältnisse war Momigliano ein Wunderkind. Zu Hause lernte er Hebräisch, Altgriechisch und Latein. An der Universität Turin studierte er Alte Geschichte. Schon zur Zeit seines Studiums spannte sich der Bogen seiner Interessen über fast alle Fächer der Altertumswissenschaft. Er führte Untersuchungen auf nahezu sämtlichen Gebieten nicht nur der klassischen, sondern auch der jüdischen Geschichte durch. Momigliano arbeitete über klassische und hellenistische, sowohl literarische als auch epigraphische, Texte, alttestamentliche Propheten, griechische Dichter oder jüdische Inschriften aus Rom. Mit 21 Jahren begann er eine Tätigkeit, die er sein Leben lang weiterführen sollte: das Rezensieren wissenschaftlicher Bücher und Artikel aus aller Welt. Allem Anschein nach waren seine intellektuellen Kräfte unbegrenzt. Schon 1934 wurde seine Monographie über Kaiser Claudius ins Englische übersetzt - ein deutliches Zeichen, daß seine Leistungen auf dem Gebiet der Alten Geschichte von einem internationalen Publikum bereits anerkannt waren. ${ }^{10}$

Einem italienischen Historiker der Generation Momiglianos konnte es natürlich nicht entgehen, daß die Geschichtsschreibung eine eigene Geschichte hatte: dies hat ihn und seine Zeitgenossen nachdrücklich Benedetto Croce gelehrt, der nicht nur eine tiefgründige allgemeine Untersuchung der Storia come pensiero e come azione (1938) geschrieben hatte, sondern sich auch intensiv mit der Analyse historischer Texte befaßt hatte, einschließlich der Scienza nuova Vicos, des Triregno Giannones und manch einer mittelalterlichen Chronik. ${ }^{11}$ Der junge Althistoriker (der mit Croce eng befreundet war und nach dem Zweiten Weltkrieg an das Croce-Institut in Neapel berufen wurde) bewunderte den alten Philosophen sehr und setzte sich mit seiner Geschichtstheorie auseinander. Schon als Student erkannte Momigliano, daß die Geschichtsschreibung immer ein fester Bestandteil der Gesellschaft und der Zeit war, aus der sie erwuchs; und auch, daß sie eine literarische Gattung war, die mindestens zum Teil mit den Mitteln der Literaturkritik verstanden werden muß.12 
Dementsprechend interessierte sich Momigliano schon in seiner Studienzeit für Texte und Themen, die sich für die spätere Entwicklung seines historischen Denkens als entscheidend erweisen sollten. 1932 veröffentlichte ein italienischer Romanist ein angeblich neues Kapitel aus Machiavellis Discorsi, in dem der florentinische Sekretär eine Stelle aus Livius anders zitierte und interpretierte, als er es im Vulgatatext seines Livius-Kommentars gemacht hatte. Momigliano wies nach, daß es sich nicht um einen neuen Text von Machiavelli handelte, sondern nur um den Versuch eines Korrektors, einen Gedächtnisfehler des Autors zu korrigieren. Zwar versuchte der Entdecker, die These Momiglianos zu widerlegen, aber es stellte sich bald heraus, daß der junge Althistoriker recht hatte. Im Verlauf der kurzen Kontroverse gelang es Momigliano zu belegen, daß er die politische Literatur der italienische Renaissance fast ebenso gut beherrschte wie die antiken Texte. ${ }^{13}$ Schon damals las er nicht nur die Neuerscheinungen der Altphilologen, sondern auch die polemischen Monographien und Rezensionsartikel, in denen Hans Baron und Ludwig Bertalot über den florentinischen Bürgerhumanismus eines Leonardo Bruni debattierten. ${ }^{14}$ Als Momigliano in den 70er Jahren über Machiavelli und Polybios schrieb, kehrte er zu diesem Interesse seiner Jugend zurück. Nach wie vor wollte er nicht nur im Sinne der modernen Philologen die Quellen und Methoden der alten Historiker rekonstruieren, sondern auch verstehen, wie Humanisten und Philosophen dieselben Texte über die Jahrhunderte hinweg interpretiert und angewandt hatten.

In gewisser Hinsicht fiel es den italienischen Gelehrten dieser Generation schwer, die Konfrontation mit der Tradition der Geschichtsschreibung zu vermeiden. Im Labyrinth der italienischen Geschichte war die kritische Geschichtsschreibung immer der Faden, der den forschenden Geist zum Zentrum oder zum Ausgang führte. Die originellsten Intellektuellen, die Helden der kritischen Vernunft, sind fast alle Historiker gewesen: Tacitus, Machiavelli, Guicciardini, Sarpi, Muratori, Maffei, Vico, Croce. Je mehr sich die politische Krise Italiens in den $20 \mathrm{er}$ und 30er Jahren verschärfte, desto dringender trat das kritische Studium der historiographischen Tradition in den Vordergrund. Nicht aus bloßer Neugier begann Momigliano schon in jüngeren Jahren, einige historiographische Probleme zu erörtern. So läßt sich auch erklären, warum andere italienische Althistoriker, wie etwa Piero Treves und Santo Mazzarino, ${ }^{15}$ sich ebenfalls daran machten, die Entwicklung ihres Faches darzustellen. 
Doch nahmen die früheren Forschungen Momiglianos die Erträge seiner Arbeit in der Nachkriegszeit nicht vorweg. Als er die Tradition der Geschichtsschreibung zu analysieren begann, widmete er sich vor allem der Untersuchung jener antiken Historiker, die als Vorläufer der modernen Geschichtsschreibung galten: er bevorzugte Thukydides gegenüber Herodot und sammelte lieber die Fragmente der verschollenen Historiker Griechenlands, als daß er die vollständigen, aber gefälschten Texte der Scriptores Historiae Augustae interpretierte. Seine Lieblingsprobleme fand er vor allem in der modernen philologischen Methode, wie sie von der deutschen Altertumswissenschaft des 19. Jahrhunderts entwickelt worden war. So versuchte Momigliano zum Beispiel, die verschiedenen Schichten des thukydideischen Geschichtswerks analytisch voneinander zu trennen, um die Arbeitsweise des Historikers entwicklungsgeschichtlich zu rekonstruieren. Diese genetische Methode, die notwendigerweise auf nicht überprüfbaren literarischen und biographischen Hypothesen beruhte, war seit dem späten 18. Jahrhundert auf Thukydides und andere Historiker angewandt worden; im Deutschland der 20er Jahre war sie besonders in Mode, weil Werner Jaeger auf sie in seinem berühmten Buch über Aristoteles (1923) zurückgegriffen hatte. ${ }^{16}$ Genau in dieser Zeit schloß sich Momigliano der deutschen Philologie an, wobei es ihm gelang, sich das ganze wissenschaftliche Handwerkszeug der Zunft anzueignen.

Auch während des politischen und kulturellen Umbruchs dieser Jahre hegte Momigliano keinen Zweifel daran, daß die deutsche Philologie den Inbegriff der Moderne in den Geisteswissenschaften darstellte. Er glaubte noch fest, daß die Historiker des späten 18. und frühen 19. Jahrhunderts eine Revolution in der Wissenschaft herbeigeführt hatten. Die Erfahrung der französischen Revolution und der romantischen Bewegung hatte den deutschen Philologen und Historikern eindringlich gezeigt, daß die Vergangenheit wirklich eine andere Welt war. Nur der Gelehrte, der imstande war, sich ganz und gar in die Lage eines griechischen Schriftstellers oder eines römischen Politikers zu versetzen, vermochte einen Text beziehungsweise den Standpunkt seines Autors historisch zu verstehen. Und nur der Historiker, der den Versuch wagte, an allen Quellen schonungslose Kritik zu üben, konnte die alte, überlieferte Geschichte durch eine wirklich moderne, kritische Darstellung ersetzen. In Festreden und Biographien beschworen die meisten deutschen Philologen immer wieder diese Prinzipien bzw. wiederholten diese Klischees. Momigliano stimmte ihnen zu, stets auf seine Weise. 
Zwar kannte Momigliano wie kein anderer die Tradition des italienischen Klassizismus, die visuelle Rhetorik Sixtus' V. und Pio Nonos sowie die literarische Ciceros und Machiavellis - dennoch bildete der karge Hellenismus von Friedrich August Wolf und Wilhelm von Humboldt sein Muster der klassischen Tradition. Er vertiefte sich in die deutsche Altertumswissenschaft, las und schrieb Rezensionsartikel und kritische Berichte zu der neuesten einschlägigen Literatur. 1936 lieferte Momigliano eine Gesamtdarstellung der Geschichtsschreibung über das römische Reich in einem Aufsatz von atemberaubender Gelehrsamkeit, in dem er nicht nur Montesquieu und Voltaire, sondern auch Bossuet und Tillemont, Vico und Gibbon behandelte. Kein deutscher Althistoriker - nicht einmal einer der emigrierten - hätte diese Texte auf vergleichbar souveräne Weise in ihrem historischen Kontext bewerten können. Dennoch verriet sich auf jeder Seite, daß der junge Historiker noch im Bannkreis der deutschen Wissenschaft stand. Er behauptete, die historische Literatur der Aufklärung, Gibbon eingeschlossen, habe die historischen Probleme des römischen Reiches nur oberflächlich zu erkennen vermocht. Erst Herder habe der alten theologischen Weltgeschichte einen neuen philosophischen Sinn gegeben. Herder sei es gelungen, das Problem des Reiches in Verbindung mit dem der Nationen zu sehen. Am Anfang war das Wort - des deutschen Denkers. $^{17}$

In einem Artikel aus dem Jahr 1935 hatte Momigliano bereits die Entwicklung der Idee des Hellenismus nachgezeichnet. Es gelang ihm auch in diesem Fall, die Texte auf neue und überzeugende Weise zu erklären. Unter anderem konnte er zeigen, daß August Böckh seinen Begriff des Hellenismus unabhängig von seinem Lehrer Wolf und seinem Schüler Droysen entwickelt hatte. ${ }^{18}$ Trotzdem beharrte Momigliano darauf, daß die Revolution von Philologie und Historie in Deutschland um 1800 stattgefunden habe. Vierzig Jahre später schrieb er, in seiner Jugend habe als unumstößliche Regel gegolten, daß man für das Studium der persischen Geschichte Griechisch können müsse, für das der griechischen Geschichte jedoch Deutsch zu lernen habe. ${ }^{19}$

In diesen Jahren nahm Momigliano ohne weiteres an (wie dies auch seine Lehrer getan hatten), daß der Entwicklungsprozeß, den die Geschichtsschreibung in ihren verschiedenen Epochen durchgemacht hatte, verhältnismäßig einfach gewesen sei. In jedem Zeitalter verkörperte eine einzelne heroische Figur die neuesten Errungenschaften der Geschichtsschreibung. Nach Thukydides, der die politische Geschichte erfunden hatte, kamen die Römer, die die Tradition der natio- 
nalen Geschichtsschreibung schufen. In der Spätantike gelang es einigen christlichen Intellektuellen, vor allem Eusebius und Augustin, das neue Genre der christlichen Geschichtsschreibung zu entwickeln; im Zeitalter der Aufklärung führten einige späte Heiden, insbesondere Voltaire und Condorcet, die Kulturgeschichte ein, usw. Zwar waren Momiglianos Lehrer geteilter Meinung darüber, ob Ranke oder Droysen der bessere Historiker war, jedoch glaubten alle fest, daß das frühe 19. Jahrhundert den Gipfel der historischen Tradition darstellte. Erst in den 40er und 50er Jahren nahm Momigliano Abstand von diesen Annahmen seiner Jugend.

Es waren vor allem die grausamen Erfahrungen der 30er Jahre und des Zweiten Weltkriegs, die Momigliano diesen Abstand ermöglichten. Im Laufe dieser dunklen Zeit nahm man ihm seine Stelle an der Universität sowie die Möglichkeit, in seinem Vaterland zu leben und zu arbeiten. Er wanderte nach England aus, wo er als »enemy alien« interniert wurde. Die begabtesten Mitglieder seiner Generation gingen ins Exil oder kamen in den Gefängnissen der Faschisten ums Leben. Schließlich starben Momiglianos Eltern in einem Konzentrationslager. Auch in England war seine persönliche Lage schwierig. Erst nach Jahren intensiver Arbeit gelang es ihm, auf Englisch frei zu sprechen und zu schreiben. Es dauerte lange, bevor er an der Bristol University eine feste Stelle erhielt. Erst Anfang der 50er Jahre wurde er auf den Lehrstuhl für Alte Geschichte am University College London berufen.

Mitten in diesen Schwierigkeiten aber lernte Momigliano allmählich neue Institutionen, Personen und Traditionen kennen, die für seine weitere Entwicklung ausschlaggebend werden sollten. Anfang der 40er Jahre erhielt Momigliano ein Stipendium, das ihm erlaubte, in Oxford zu leben und zu arbeiten. Dies gab ihm reichlich Gelegenheit, in der Bodleian Library zu forschen, zumal in den Sammlungen der großen Gelehrten des 16. und 17. Jahrhunderts, wie etwa denen von John Selden und Isaac Casaubon. Die Traditionen der humanistischen Gelehrsamkeit, die von den Handschriften und Folianten der Bodleian Library ausströmten, erschienen ihm in der einzigartigen Atmosphäre Oxfords sehr lebendig. Momigliano beschwor immer gern »die posthume Gesellschaft des Casaubonus", die er so oft winmitten seiner Bücher und Handschriften in Bodley's Library« genossen hatte. ${ }^{20}$

In Oxford kam Momigliano auch in Kontakt mit Forschern, die sich auf neue Gebiete der Kulturgeschichte spezialisiert hatten und in der Lage waren, ihm neue methodologische Wege zu weisen. So lernte er Beryl Smalley kennen, eine Mediävistin, die sich auf dem Gebiet der 
mittelalterlichen Schulen und Universitäten außerordentlich gut auskannte und Pionierarbeit mit ihrer Geschichte des Bibelstudiums geleistet hatte. Sie schloß Freundschaft mit Momigliano, verbesserte seine ersten englischen Schriften und trug viel dazu bei, seine Aufmerksamkeit auf Probleme der Überlieferung klassischer Texte im Mittelalter und in der Renaissance zu lenken. Momigliano war bereits mit den Arbeiten des Münchener Paläographen Ludwig Traube vertraut, der versucht hatte, die Handschriftenforschung zu einer Art interdisziplinärer Kulturgeschichte zu machen; seine Freundschaft mit Smalley hatte zur Folge, daß er auch von der englischen Ausprägung dieser Disziplin, die auf eine spezifische Verbindung von Ideen- und Bildungsgeschichte hinauslief, Kenntnis nahm. ${ }^{21}$

Noch wichtiger wurde für Momigliano das Warburg Institute in London. Hier fand er ein Forschungszentrum, das nicht nur von Kunsthistorikern, sondern auch von Philologen und Historikern stark frequentiert wurde. Schon in den 20er und 30er Jahren war das Institut, damals in Hamburg, der einzige Ort in Europa gewesen, an dem das Nachleben der Antike das zentrale Thema einer ganzen Reihe von berühmten Seminaren und Vorträgen gebildet hatte. Im Londoner Institut stand Momigliano in einem regen Austausch mit Fritz Saxl und Gertrud Bing, Frances Yates und Donald Gordon - mit Gelehrten also, die Italien und Deutschland kannten, die die gesamte Humanismus-Tradition als ihr besonderes Forschungsfeld betrachteten und die Momigliano am Institut in einer Weise willkommen hießen, wie dies bei den englischen Althistorikern in Oxford und Cambridge nicht der Fall gewesen war.

Das Warburg Institute stand zum Teil in der Tradition der deutschen Philologie. Nicht nur der große Kulturhistoriker Jacob Burckhardt, der - nach dem Verdikt von Wilamowitz - »für die Wissenschaft nicht existiert", sondern auch der große Religionshistoriker Hermann Usener hatte Aby Warburg in methodischer Hinsicht stark beeinflußt. ${ }^{22}$ In den $20 \mathrm{er}$ Jahren hielten Rudolf Pfeiffer und Eduard Fraenkel in Hamburg wichtige Vorträge, die im Laufe der Zeit vom Institut veröffentlicht wurden. ${ }^{23}$ Später wurde das wiedereröffnete Londoner Warburg Institute zu einer der wichtigsten Begegnungsstätten für ausgewanderte Philologen, denen Saxl mit Rat und Tat zur Seite stand. Dennoch stellte die Arbeit der Gelehrten im Kreis des Instituts einen neuen Ansatz zur Geschichte der klassischen Tradition dar, demzufolge die Dichter des Mittelalters und die Humanisten der Renaissance wesentliche Kunstformen und Traditionen der Antike, wie etwa die 
Ironie des Lukian und das Pathos des Lukan, nicht nur rezipiert und überliefert, sondern auch tiefgründig interpretiert hatten, und zwar bevor sich die Philologenzunft an die Arbeit machte, dieselben Elemente als wichtige Bestandteile der klassischen Tradition zu betrachten. Am Warburg Institute ging es nicht mehr nur darum zu beweisen, daß die deutschen Philologen es ermöglicht hatten, klassische Texte und Denkmäler historisch zu verstehen, sondern mehr noch zu verstehen, wie mittelalterliche und frühmoderne Humanisten und Antiquare, Dichter und Künstler kreativ und einfühlsam mit Texten und Bildern umgegangen waren. Warburg und Saxl fanden, daß die philologische Leistungsbilanz der Humanisten erheblich besser ausfiel, als die meisten Philologen anzunehmen pflegten.

In der Krise der 40er Jahre räumte Momigliano ohne weiteres ein, daß die Traditionen der deutschen Philologie und Geschichtsschreibung in verschiedener Hinsicht problematisch waren. Schon vor dem Weltkrieg war er fest davon überzeugt, daß der unpolitische »dritte $\mathrm{Hu}$ manismus« Werner Jaegers mehr ein Symptom des Verfalls der deutschen Wissenschaft war, als daß er ein Heilmittel dagegen darstellte. Natürlich verachtete er die Althistoriker und Philologen, die Stellen an Instituten für Rassenkunde bekleideten und deren Arbeiten die Annahmen und Vorurteile der Nazis widerspiegelten. Nach dem Krieg empfand Momigliano immer wieder Abscheu, wenn wissenschaftliche Koryphäen Deutschlands und Italiens so taten, als ob große Historiker und Philologen nicht ins Exil getrieben worden oder ums Leben gekommen wären. Die Entideologisierung der Alten Geschichte und der Klassischen Philologie, die in den 50er Jahren stattfand, hat Momigliano als eine Gefahr wahrgenommen: wer die Vergangenheit nicht in Erinnerung hielt, lief Gefahr, sie zu wiederholen. So nahm Momigliano die italienische Übersetzung der Griechischen Geschichte Helmut Berves zum Anlaß, die Kehrseite der Karriere des Autors in einer verheerenden Rezension zu entlarven, die jüngere Historiker in Deutschland und anderswo wiederum dazu anregte, die ganze Geschichte der Altertumswissenschaft im NS-Staat zu erforschen. Althistoriker, so Momigliano, waren es auch den Opfern dieser Zeit schuldig, die neuere Geschichte ihres Faches zu rekonstruieren. Das Studium der historischen Tradition stellte seines Erachtens kein Hobby dar, das besonders gut dafür geeignet war, in Festreden von emeritierten Professoren ausgeübt zu werden, sondern einen Schlüssel, der seinem Besitzer die Möglichkeit gab, die intellektuellen Untaten und institutionellen Verstrickungen seiner Vorgänger historisch zu verstehen, ohne sie einfach zu relativieren. ${ }^{24}$ 
Tatsache ist aber, daß sich Momigliano schon damals mehr für die frühere als für die neueste Geschichte seines Faches interessierte. In den 40er Jahren vertrat er in einem am Warburg Institute gehaltenen Vortrag über Friedrich Creuzer eine neue methodologische These. Momigliano behauptete, daß nur derjenige Historiker das Fach wieder aufbauen könne, der konsequent versuchte, sich in die vorangegangene Geschichte seines Faches zu vertiefen, und dabei wichtige und zu Unrecht vergessene Probleme und Kontroversen zutage fördern könne. Geschichte und Zukunft des Faches seien eng mit einander verknüpft. Die Entwicklung der Wissenschaft stellte seiner Meinung nach keine einfache Vorwärtsbewegung dar. Einerseits waren die Gründe, die einen bestimmten Text oder ein bestimmtes Problem in den Vordergrund rückten, des öfteren eher politischer, literarischer oder sogar religiöser als rein wissenschaftlicher Natur. Andererseits hatten auch die originellsten Philologen oft auf die Themen und Methoden früherer Forscher zurückgegriffen, um eigene Theorien zu entwickeln. ${ }^{25}$

Aus diesem Tatbestand zog Momigliano Konsequenzen. Zwar räumte er ein, daß es immer wieder bahnbrechende Individuen und Bücher gegeben habe. Dennoch durfte der Wissenschaftshistoriker nie ohne weiteres davon ausgehen, daß eine bestimmte Edition oder ein bestimmter Kommentar einen Bruch mit der älteren wissenschaftlichen Tradition dargestellt habe, bevor er nicht den ganzen historischen Kontext und die ganze ältere Literatur in Betracht gezogen hatte. Sonst würde er unvermeidlich Gefahr laufen, das Neue an einem wissenschaftlichen Buch mit dem Überlieferten zu verwechseln oder die politischen Gründe, aus denen ein Gelehrter eine methodologische Entscheidung traf, außer acht zu lassen. So stellte sich heraus, daß der Historiker der klassischen Philologie genauso unvoreingenommen und interdisziplinär vorgehen mußte, wie der Altertumswissenschaftler selbst - allerdings nicht der durchschnittliche Altertumswissenschaftler, der behaglich in einem deutschen Seminar oder in einer englischen Universität lehrt und normale, fachspezifische Projekte durchführt, ohne sich je die Frage zu stellen, wie es dazu gekommen ist, daß er genau dieses oder jenes Problem untersucht. Nach Momiglianos Auffassung vermochte wirklich nur der ideale Altertumswissenschaftler, wie ihn sich Wolf oder Böckh vorgestellt hatten, der Geschichte des Faches nachzugehen. Die Geschichte der Altertumswissenschaft stellte kein Thema für emeritierte Altphilologen und Hobby-Wissenschaftshistoriker dar, sie bot jedoch den wenigen Spezialisten ein weites Feld für neue Forschungen. Auf der Grundlage dieser einfachen, 
aber anspruchsvollen Prinzipien gelang es Momigliano, eine Art Forschungsprogramm aufzustellen, das er in den nächsten vierzig Jahren durchführen sollte, nicht nur in seinen Artikeln, sondern auch in den Seminaren und Vorlesungen, die er am Warburg Institute, an der Scuola Normale di Pisa, und an der University of Chicago hielt.

Momigliano hörte nie auf, Probleme der jüdischen, griechischen und römischen Geschichte zu untersuchen. Er befaßte sich weiterhin - wie sich im ersten Band dieser Ausgabe deutlich zeigt - mit der Entwicklung der Geschichtsschreibung in der Antike. Dennoch begnügte er sich keineswegs mit der langen Reihe bahnbrechender Studien zu jüdischen, griechischen und römischen Historikern, in denen es ihm u.a. gelang, mit dem alten Klischee aufzuräumen, daß die Griechen ein zyklisches und die Juden ein lineares Geschichtsbild gehabt hätten, sondern er machte sich auch daran, die Entwicklung der frührömischen Gesellschaft und Verfassung sowie die Christianisierung der spätrömischen Aristokratie nachzuzeichnen. Die politischen und kulturellen Begegnungen und Konflikte von Juden, Griechen und Römern übten immer noch einen besonderen Reiz auf Momigliano aus. Hinzu kamen kontinuierlich neue Themen und Interessen. In jüngeren Jahren hatte Momigliano kein großes Interesse für die französische Literatur und Kultur gezeigt; seit den 50er Jahren las er eifrig sowohl die angeblich "neuen Historiker « als auch die Philosophen und Anthropologen, die behaupteten, eine Revolution in den Geisteswissenschaften herbeigeführt zu haben. Er versuchte, den neuen Methoden Jean-Pierre Vernants und Michel Foucaults Rechnung zu tragen, wobei er auch, bekanntlich aus sehr unterschiedlichen Gründen, stark gegen Georges Dumézil und Pierre Vidal-Naquet polemisierte.

Die Geschichte der klassischen Philologie zog sich wie ein roter Faden durch die Vielfalt der späteren Schriften Momiglianos, obwohl sie sich in verschiedenen Formen niederschlug. Momigliano spürte gern den Schicksalen einzelner Texte nach, was ihm zu zeigen ermöglichte, wie das historisch-politische Denken der Neuzeit aus den Traditionen der humanistischen Gelehrsamkeit erwachsen war. Die Texte, die ihn am meisten interessierten, spielten jeweils eine verwickelte Rolle in dieser Entwicklung. Tacitus war nicht nur das große Beispiel dafür, wie ein Herrscher oder ein Höfling politische Ereignisse realistisch interpretieren sollte, sondern offenbarte auch die Geheimnisse alter - und moderner - Tyrannen, was ihn zur Lieblingslektüre aller Revolutionäre machte. In einem Aufsatz aus den späten 40er Jahren, der von atemberaubender Gelehrsamkeit zeugt, analysierte Momigliano 
den europäischen Tacitismus, wobei es ihm gelang, den originellsten Tacitisten - wie Marc-Antoine Muret und Justus Lipsius - gerecht zu werden sowie den fast unbekannten Carolus Paschasius als denjenigen Denker zu identifizieren, der die politische Bedeutung der taciteischen Texte zum ersten Mal in einem Kommentar explizit dargelegt hatte. Nicht Lipsius, sondern Paschasius machte Momigliano dafür verantwortlich, daß diese »diskrete Bewegung der Gelehrsamkeit in Italien explodierte«. ${ }^{26}$ Später, Mitte der 70er Jahre, kehrte Momigliano zu Machiavelli in einem Aufsatz zurück, in dem er ausführlich zeigte, daß Polybios im Florenz des frühen 15. Jahrhunderts vor allem als Historiker gegolten hatte. Leonardo Bruni versuchte sogar zu erklären, inwiefern Polybios und andere griechische Historiker »von ihren eigenen Quellen bedingt waren « - ein Verfahren, das Momigliano als »den wirklichen Beginn der historischen Kritik« bezeichnete. Erst hundert Jahre später wurde der griechische Historiker in derselben Stadt von Machiavelli als politischer Denker wiederentdeckt - und noch später, im Zeitalter der Heeresreform der Oranier, sollte er einen guten Ruf »als Militär- und Diplomatie- Historiker" genießen. ${ }^{27}$ So wurde Rezeptionsgeschichte zu einer neuen Art von Geistesgeschichte.

Momigliano trug nicht nur den bewegten Schicksalen der klassischen Autoren in den sich wandelnden Nachwirkungen, sondern auch den Biographien und Methoden der modernen Forscher Rechnung. Vor allem in den 40er Jahren, als er die Schätze der Bodleian Library und der Bibliothek Warburg durchforschte, hat er wahrnehmen müssen, daß es nicht die deutschen Historiker um 1800, sondern die sogenannten "Antiquare in der alten wie in der modernen Welt gewesen waren, die als erste den Versuch gewagt hatten, die verschollenen Institutionen und Rituale, den Glauben und die Sitten vergangener Gesellschaften aus bruchstückhaft erhaltenen Dokumenten zu rekonstruieren. Die Antiquare nahmen nach Momiglianos Ansicht viele Ergebnisse der modernen Geschichtswissenschaft vorweg, indem sie konsequent versuchten, eine streng wissenschaftliche Kritik zu entwickeln, wobei sie auch Pionierarbeit in der Sammlung und Bewertung von Inschriften und anderen materiellen Überresten der Antike und des Mittelalters leisteten. Nach wie vor stellt Momiglianos Aufsatz »Ancient History and the Antiquarian« von 1950 eine unentbehrliche Synthese dar, in der nicht nur die allgemeine Entwicklung der Geschichtswissenschaften in der frühen Neuzeit, sondern auch die Leistungen etlicher Antiquare ausführlich behandelt werden. ${ }^{28}$ In späteren Studien untersuchte er noch ausführlicher die Karrieren und Ar- 
beiten der großen Paläographen und Diplomatiker Mabillon und Montfaucon. ${ }^{29}$

Momigliano vergaß nie, daß Institutionen eine große Rolle in der Geschichte der Geisteswissenschaften gespielt haben. So widmete er sich in dem großen Aufsatz von 1950 den italienischen Akademien, die im Zeitalter der Aufklärung der historischen und vor allem der archäologischen Forschung neue Impulse gaben. In einem seiner spätesten Aufsätzen erklärte er, warum die Geschichte so lange »aus dem Lehrplan ferngehalten wurde« - auch noch, nachdem die klassische Rhetorik wieder zum zentralen Bildungsgut der Schulen geworden war - und warum die deutschen Universitäten eher als die italienischen zum ersten Mal der Geschichte als Disziplin einen bestimmten, wenn auch noch schwankenden Platz im Lehrplan zuwiesen. Hier wurde exemplarisch gezeigt, wie der Wissenschaftshistoriker die komplizierte Wechselwirkung zwischen Forschung und Lehre auf der einen Seite und dem institutionellen bzw. kulturellen Kontext auf der anderen klarstellen sollte. ${ }^{30}$

Niemand wußte besser als Momigliano, daß die Geschichte in der Antike ebenso wie in der frühen Neuzeit einen Bestandteil der rhetorischen Tradition bzw. eine literarische Gattung dargestellt hatte. Ihm gelang es immer wieder, den Stil eines großen Historikers treffend zu charakterisieren. Dennoch bestand er prinzipiell darauf, daß die Geschichtsschreibung auf Forschung basieren muß. Die neue Historik seines Freundes Hayden White empfand er als eine Provokation, die ihm Anlaß gab, die Aufgabe des Historikers auf seine eigene Weise zu definieren. ${ }^{31}$ Auch historiographisch hatte er schon über die Jahre hinweg klarzustellen versucht, wie in jeder Geschichtsschreibung Stil und Gelehrsamkeit, Rhetorik und Forschung zusammenkommen mußten. Schon 1954 zeigte Momigliano, daß Gibbon, der gewiß ein Meisterwerk der englischen Prosa geschrieben hat, das besondere Verdienst zukam, gelehrte sowie philosophische Probleme in einem Zusammenhang erörtert zu haben - d.h., daß er neue analytische Möglichkeiten für die Historiographie entwickelt hatte. ${ }^{32}$

Momigliano befaßte sich am liebsten mit Individuen: zum Beispiel mit Vico, dessen skurrile Kombination von brillanten Fehldeutungen und schlichten Fehlern in der Textinterpretation er äußerst irritierend fand, oder auch mit Cassiodor, den er als einen der "geringeren Männer« sehr schätzte, »die zu bewahren versuchten, was bewahrt werden konnte, und die die Aufgabe des elementaren Unterrichts nicht verschmähten, als elementares Unterrichten vonnöten war«, oder auch 
mit Gibbon. ${ }^{33}$ Momiglianos Essays bieten dem Leser ausgezeichnete Beispiele einer kritischen Geschichte der Geisteswissenschaften, die die Quellen einer strengen Kritik unterzieht, die immer konsequent interdisziplinär vorgeht und die immer wieder neue Erträge hervorbringt. Sie können aber genausogut als literarische Porträts genossen werden, die in ihrer einzigartigen Verbindung beispielloser Gelehrsamkeit und gewandten Stils ein Musterbeispiel traditioneller Geschichtsschreibung darstellen und gleichzeitig mit ihrer psychologischen und historischen Einfühlung die historischen Erfahrungen eines großen Denkers und Schriftstellers widerspiegeln.

Die Essays in diesem Band sind nach den in den Contributi gedruckten Texten übersetzt worden. Die Übersetzer haben die Zitierweisen der antiken Quellen nach der Liste der Autoren und Werktitel im »Neuen Pauly« weitgehend vereinheitlicht, wobei sie offensichtliche Fehler stillschweigend verbessert und unvollständige Literaturangaben ergänzt haben. Die den Aufsätzen vorangestellten knappen Einführungen stammen vom Herausgeber dieses Bandes. ${ }^{34}$ 